\title{
La construcción social de los mercados de agua en Chile: un enfoque desde la geografía legal
}

\section{The social construction of water markets in Chile: an approach from the legal geography}

\author{
Chloé Nicolas Artero'
}

\begin{abstract}
RESUMEN
El artículo propone un enfoque desde la geografía legal para entender la construcción social de los mercados de agua en la cuenca semiárida del río Elqui, desde la segundad mitad del siglo XX. A partir de metodologías cualitativas (etnografía, entrevistas, archivos) revela la dimensión conjuntamente espacial y jurídica de la formación de los mercados de agua. Los objetos técnicos hídricos instalados desde la segunda mitad del siglo XX atraen nuevos inversionistas y transforman la economía política del valle. Desde entonces, los usuarios usan los objetos técnicos hídricos e instrumentalizan o producen derecho a escala local para comprar, vender o alquilar derechos de agua, formando cinco mercados de agua.
\end{abstract}

Palabras clave: Mercado de agua, Objetos técnicos, Geografía legal, Semiárido, Chile

\begin{abstract}
The article proposes an approach from the legal geography to understand the social construction of water markets in the semi-arid basin of the Elqui river, from the second half of the 20th century. Using qualitative methodologies (ethnography, interviews, archives), it reveals the jointly spatial and legal dimension of the formation of water markets. The technical water objects installed since the second half of the 20th century attract new investors and transform the political economy of the valley. Since then, the users have used technical water objects and have instrumentalized or produced law locally to buy, sell or rent water rights, forming five water markets.
\end{abstract}

Keywords: Water Markets, Technical objects, Legal Geography, Semiarid, Chile 
Durante la Conferencia de Dublín de 1992, los recursos hídricos fueron reconocidos como un bien económico. Desde entonces distintas instituciones internacionales han promovido la implementación de mercados de agua. Varios países, como los estados occidentales de los Estados Unidos, Australia, España y Chile han incorporado mecanismos de mercado en su legislación hídrica. Definimos los mercados de agua como los intercambios mercantiles de derechos de agua formales o informales entre actores. Los derechos de agua formales son aquellos que han sido regularizados reconociendo el derecho de propiedad privada sobre ellos, así como su libre transferibilidad entre usuarios (Briscoe, 1998; Vergara Blanco, 2015; Molle, 2004). En teoría, la asignación de los derechos de agua por el mercado debería incentivar a los actores a mejorar la eficiencia en su uso, es decir ahorrar el recurso escaso. Los intercambios informales consisten en vender volúmenes de agua. En ciertas ocasiones se denominan alquileres de derechos de agua ${ }^{2}$.

La literatura sobre los mercados de agua es abundante. Muchos trabajos adoptan un enfoque económico para analizar su eficiencia: los investigadores evalúan sus condiciones de emergencia mediante la definición de los precios y la reducción de los costos de transacción (Easter et al., 1999; Saliba, 1987; Donoso et al., 2000; Bjornlund, 2003; Hadjigeorgalis, 2004; 2009). Un segundo enfoque institucionalista considera el mercado de aguas como una institución social e histórica (Howitt, 1994; Petit, 2004). Este no surgiría naturalmente, sino que por una superposición de prácticas sociales, decisiones políticas y reglas que se reproducen en el tiempo (Yáñez \& Lorenzo, 2003). Por lo tanto, las decisiones políticas y las disposiciones legales que reconocen la propiedad privada sobre las aguas participan a la formación de un mercado de aguas (Casado-Pérez, 2016). A nivel local, las instituciones siguen siendo esenciales para reducir los costos de transacción y garantizar el encuentro entre la oferta y la demanda (Reis, 2014; Grafton et al., 2011; Garrick et al., 2018). Sin embargo, esta afirmación es criticada por antropólogos que señalan la existencia de la venta de derechos de uso temporal por parte de los turneros de agua en las comunidades de regantes (Bedoucha, 1987). Estos arreglos y compromisos permiten la viabilidad del sistema de reparto de las aguas en ausencia de intervención estatal y de un marco regulatorio local.

El caso chileno ha despertado un gran interés en las ciencias sociales. En la década de los años ochenta, la Ley №2603 proclamada en 1979, la Constitución de 1980 y el Código de aguas de 1981 establecen un marco jurídico que reconoce la libre disposición, goce y uso de los derechos de agua, disociados de los derechos de uso del suelo. Las primeras publicaciones sobre este tema subrayan el supuesto éxito de los mercados de agua: habrían permitido el auge de las exportaciones y el desarrollo económico del país (Rosegrant \& Binswanger, 1994; Rosegrant \& Gazmuri, 1995; Rios \& Quiroz 1995, Thobani, 1997). Sin embargo, estas investigaciones han sido objeto de fuertes críticas por no basarse en análisis empíricos. Otras generalizan el caso excepcional de la cuenca del río Limarí (Hearne \& Easter, 1997) donde el mercado es muy activo debido a la presencia de infraestructuras y de una junta de vigilancia ${ }^{3}$ que facilita las transacciones reduciendo sus costos.

Investigaciones más recientes han mostrado que estos trabajos defienden posiciones ideológicas para legitimar el pensamiento de la nueva economía de los recursos naturales (Dourojeanni

No son declaradas ni inscritas en el registro de propiedad de los CBR (Conservador de Bienes Raíces).

Organización encargada de la distribución de las aguas superficiales, encabezada por un directorio elegido por los dueños de derechos de agua de la cuenca. 
\& Jouravlev, 1999; Petit, 2004; Bauer, 1997, 2015; Prieto, 2015). Mediante investigaciones empíricas en diferentes cuencas del país revelan, en varios casos, la ausencia de compra y venta de derechos de agua. Según el geógrafo Carl Bauer (2004), varios factores explican esta situación. Por una parte, la geomorfología de los ríos chilenos y la rigidez de la infraestructura de riego impiden los trasvases de agua. Por otra parte, la insuficiente seguridad jurídica sobre los derechos de agua y la resistencia cultural frenan las ventas de los derechos de agua. Además, la ausencia de señales de precios y el alto valor de los títulos de agua en comparación con las opciones menos costosas, como, por ejemplo, mediante la solicitud gratuita de derechos subterráneos a la Direccion General de Aguas o la construcción de pozos ilegales, explican los pocos intercambios formales. Estos últimos argumentos han sido confirmados por una investigación de Jessica Budds (2012) realizada en la cuenca de La Ligua. Destaca el trabajo de Manuel Prieto (2016) que revela la existencia de un mercado de aguas dentro de una comunidad indígena atacameña. Esta estableció un fondo colectivo de agua comprando derechos a algunos de sus miembros para evitar su adquisición por las empresas mineras.

Si la mayoría de los trabajos recientes concluyen en la casi-inexistencia de los mercados de agua, este artículo propone analizar la dimensión legal y espacial de su construcción social a escala de una cuenca. Sin embargo, no se retoman los enfoques economicistas o neo-institucionalistas: mostramos como los mercados de agua son producidos por la reapropiación conjunta y estratégica, por los usuarios, del derecho y del espacio - a través de los objetos técnicos hídricos -. Estos suelen ser introducidos para facilitar la captación, acumulación y circulación del agua potable o de los recursos hídricos y producir así un mejoramiento técnico sin tomar necesariamente en consideración su impacto en los sistemas hidrosociales (Germaine et al. 2019). Discutida por los trabajos de la Science and Technology Studies (Latour 1991, Callon 1986) y de la Political Ecology (Obertreis et al., 2016), la noción de objetos técnicos (Akrich, 1987) permite subraya como justamente estas infraestructuras y dispositivos tecnológicos reconfiguran las relaciones de los seres humanos entre sí y con la naturaleza y tienen por ello una dimensión política. Para analizar la dimensión jurídica del uso de estos objetos, utilizamos elementos conceptuales y metodológicos de la geografía legal crítica (Holder \& Harrison, 2003; Braverman et al., 2014). Esta corriente de la geografía cuestiona las relaciones entre legalidad, espacialidad y poder. Según Nicholas Blomley (1994), este enfoque no debe limitarse a documentar estas relaciones que inducen, según él, su naturalización y despolitización. En efecto, en geografía se suelen analizar el derecho y el espacio para comprender los efectos del uno sobre el otro, conduciendo a su reificación en campos separados, simplificando la complejidad de sus relaciones. En nuestro caso, este enfoque teórico permite analizar la instrumentalización cotidiana del espacio y del derecho por los usuarios de una cuenca para acceder al agua, y como esta, produce distintos mercados de agua. ¿En qué medida la apropiación conjunta del derecho y del espacio participa a la construcción social de los mercados de agua en una cuenca?

Este artículo persigue varios objetivos. El principal es describir la dimensión jurídica y espacial de la construcción de los mercados de agua. Para ello, por una parte, propone identificar los principales objetos técnicos hídricos del valle. La gran mayoría han sido instalados a partir de la segundad mitad del siglo XX, transformando la economía política de la cuenca. Por otra parte, busca describir la instrumentalización local del derecho y de los objetos técnicos hídricos que realizan los actores para intercambiarse el agua. Esto permitirá, identificar distintos mercados de agua en la cuenca. 


\section{La cuenca del río Elqui en la región semiárida de Coquimbo}

El estudio de caso se centra en la cuenca del río Elqui ubicada en el norte de la región semiárida de Coquimbo. La cuenca exorreica del río Elqui se extiende desde la Cordillera hasta el Océano Pacífico. Cubre un área de $9,794 \mathrm{~km}^{2}$ desde las tierras de altas montañas y sus numerosos glaciares y glaciares rocosos hasta su desembocadura en la bahía de Coquimbo. Sin embargo, confinada entre cadenas de montañas medianas y bajas, la planicie aluvial es mucho menos extensa. Abarca las tierras de regadío y los principales asentamientos que constituyen el área de estudio. Por lo tanto, éste se limita al valle colindante al río Elqui $(75 \mathrm{~km})$ y sus dos afluentes principales, el río Turbio $(40 \mathrm{~km})$ y el río Derecho $(65 \mathrm{~km})$. Ambos convergen en Rivadavia, un pueblo situado a una altitud de $815 \mathrm{~m}$. La pendiente altitudinal del valle varía, en una sección de aproximadamente $170 \mathrm{~km}$, entre $0 \mathrm{~m}$ de altitud al nivel de la playa de La Serena y $6.255 \mathrm{~m}$ de altitud máxima en la cima de la montaña de los Olivares. El régimen del rio es de tipo nival: su caudal aumenta en primavera durante el deshielo, de noviembre a enero. La precipitación media anual en La Serena es de $90 \mathrm{~mm}$. Las precipitaciones se concentran en períodos cortos entre abril y mayo.

En 1997, Robert Hearne y William Easter compararon los mercados de agua de cuatro cuencas chilenas mediante una encuesta por cuestionario. En el valle del río Elqui, los agricultores entrevistados declararon establecer pocas transacciones formales ${ }^{4}$. Muchas veces, fueron compras de derechos de agua de papel, es decir, cuya agua no era efectivamente utilizada por su propietario. Los autores concluyeron en la inexistencia de un mercado de aguas en la cuenca del río Elqui. Sin embargo, los registros de propiedad en los Conservadores de Bienes Raíces (CBR) de La Serena y de Vicuña muestran la existencia de una multitud de intercambios de derechos de agua formales entre 1950 y 2016, con un auge importante desde el año 1981 (Figura №1). La contradicción entre las conclusiones de la investigación realizada en 1997 y los registros de los CBR muestran la necesidad de estudiar la construcción social de los mercados de agua desde un nuevo enfoque teórico y metodológico. 
Figura N¹.

Inscripciones de ventas e hipotecas de derechos de agua en la cuenca de Elqui entre 1950 y 2016

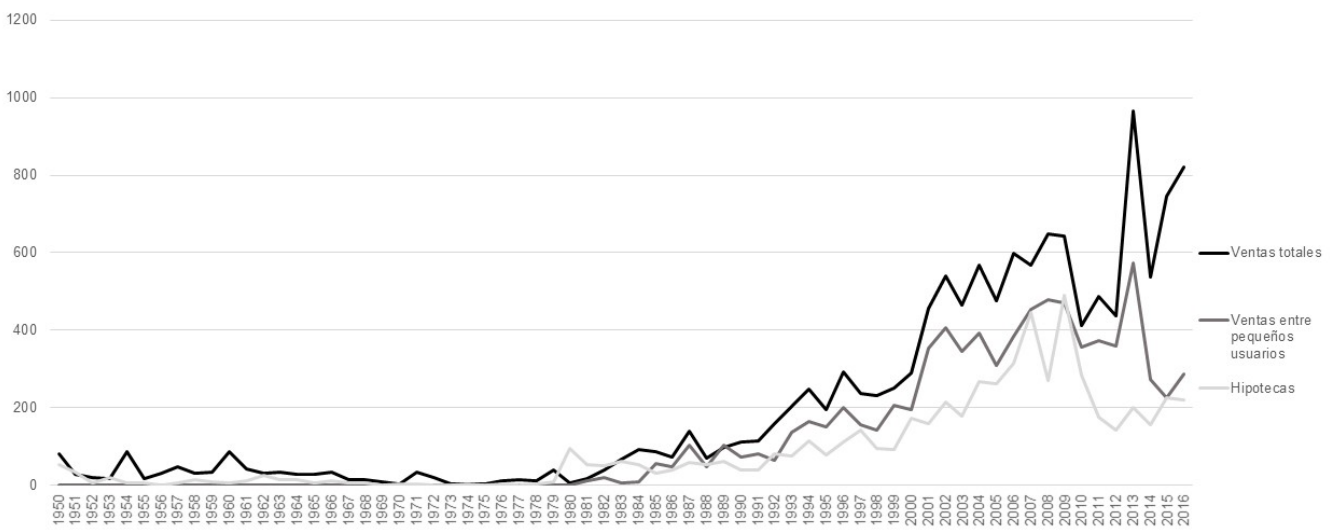

Fuente: Elaboración propia. CBR de Vicuña y La Serena, 1950 - 2016.

En el valle de Elqui, el aumento de los intercambios formales de derechos de agua a partir de la década del 2000 puede explicarse por varios factores. Por un lado, desde esa fecha la Dirección General de Agua (DGA) ha dejado de conceder derechos sobre las aguas subterráneas y superficiales al establecer áreas de restricción en la cuenca. Antes de esa fecha, era más rentable solicitar a la DGA derechos de agua permanentes o temporales otorgados gratuitamente, o construir pozos ilegales. Por otro lado, desde la construcción del embalse Puclaro en 1995, el Ministerio de Obras Publicas ha financiado programas para regularizar los derechos de agua, teniendo como consecuencia su inscripción en los CBR de forma separada de los títulos de propiedad sobre el suelo. Esa formalización favoreció los intercambios de derechos de agua. Sin embargo, varias preguntas permanecen sin respuesta. ¿Cómo explicar las transacciones formales existentes antes de la década del 2000? ¿Existen, en la cuenca, intercambios de derechos informales o de papel?

\section{Metodología de investigación}

Para identificar los intercambios formales e informales se utilizan cuatro métodos de investigación cualitativos que persiguen distintos objetivos. En primer lugar, se reconstruye la historia de los objetos técnicos hídricos del valle y la transformación de su economía política a partir de la segunda mitad del siglo XX. Este trabajo ha implicado analizar fuentes históricas consultando los archivos nacionales del Ministerio de Obras Públicas y de la Dirección de Obras Hidráulicas (DOH), así como la base de datos de los proyectos asociados a la ley de riego aplicada por la Comisión Nacional de Riego (CNR) y al programa de agua potable rural implementado por la $\mathrm{DOH}$. Además, para describir la evolución de los intercambios formales de derechos de agua, se han consultado los registros de propiedad de los CBR de Vicuña y de La Serena, entre 1950 y 2016. En 
estos aparecen los nombres y direcciones de los titulares, la cantidad de derechos que venden o adquieren, la ubicación del punto de captación de agua, así como la cantidad, fecha y lugar de la transacción.

En un segundo lugar, esta investigación descansa sobre un trabajo etnográfico que permite identificar las apropiaciones conjuntas y estratégicas del derecho y de los objetos técnicos hídricos por los actores para vender o comprar agua. Este trabajo se basa en la realización de múltiples observaciones y conversaciones con los habitantes durante tres inmersiones prolongadas en el valle. La recopilación de datos se ha desarrollado durante nueve meses en un período de tres años consecutivos, del año 2016 al 2018. Las observaciones y los fragmentos de conversaciones escuchados han sido transcritos en tres cuadernos de campo, sumando más de 300 páginas de notas.

Finalmente, esta investigación se basa en la realización de 120 entrevistas semiestructuradas con diversos actores de la cuenca: los dirigentes de las juntas de vigilancia del río Elqui y del Estero Derecho, los agricultores, los representantes de las empresas inmobiliarias, el operador de agua potable Aguas del Valle SA, la empresa minera San Gerónimo y un representante del Ministerio de Minería. El protocolo de la investigación se basa en la implementación de una guía de entrevista adaptada para cada tipo de actores. Todos los datos recopilados a través de estas tres metodologías de investigación han sido codificados y analizados en el programa ATLAS.ti.

\section{Los objetos técnicos hídricos en la transformación espacial del valle}

Desde la segunda mitad del siglo XX, el Estado chileno ha instalado objetos técnicos hídricos en el valle. Estos facilitan la captación, acumulación y la circulación del agua potable o de los recursos hídricos, como son por ejemplo los tanques, los canales y sus compuertas, las bombas o los pozos. La instalación de estos objetos facilita las inversiones y atrae nuevos actores en el valle lo que transforma su economía política (Figura $\mathrm{N}^{\circ} 2$ ). 
Figura $\mathrm{N}^{\circ} 2$.

Nuevos objetos técnicos hídricos y producción del espacio 1950 - 2018
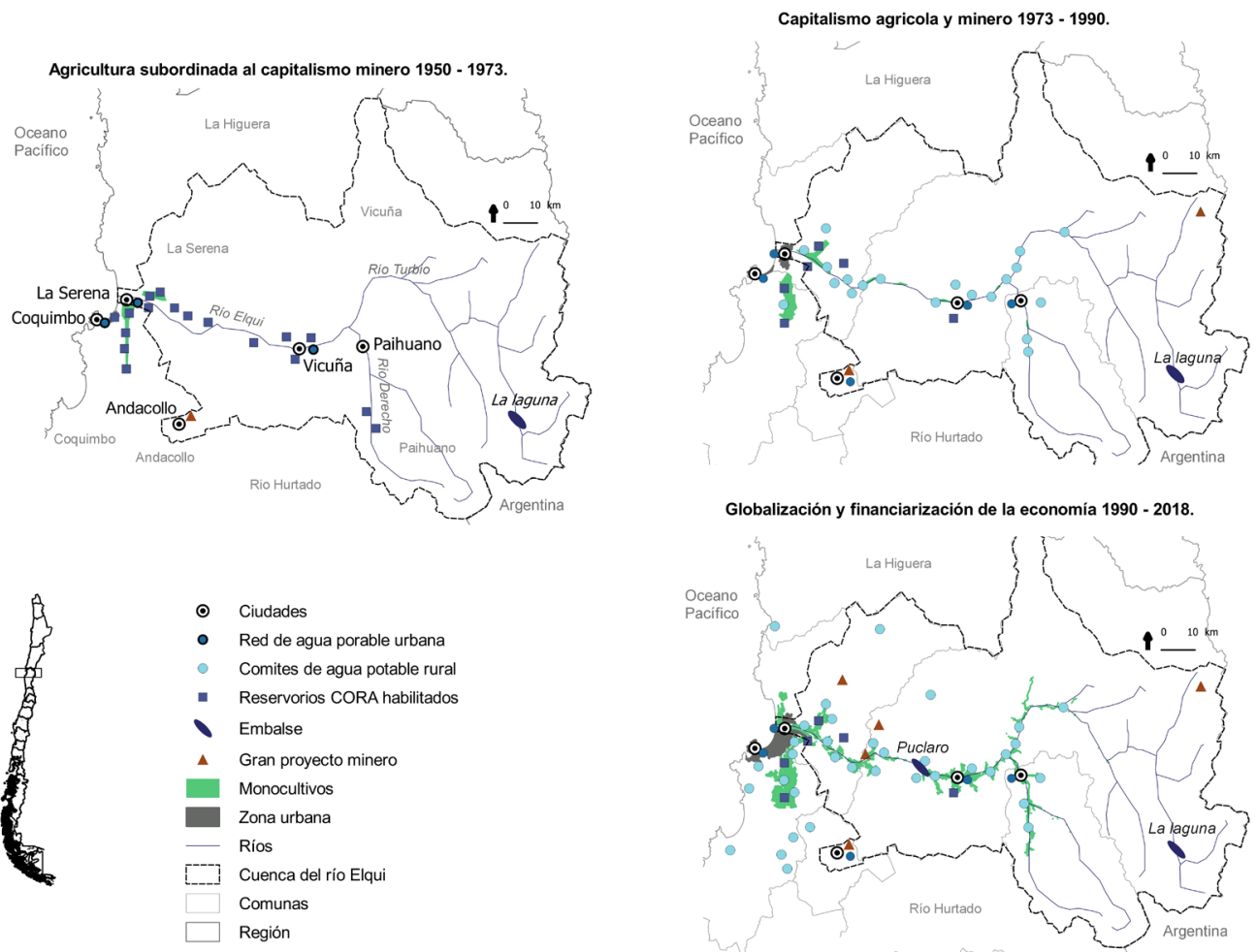

Fuente: Elaboración propia. Base de datos CNR, DOH.

\section{Tanques de agua para la agricultura y el capitalismo minero del norte (1945 - 1973)}

Entre 1945 y 1973, las inversiones en objetos técnicos hídricos permitieron aumentar la producción agrícola destinada a satisfacer la demanda del capitalismo minero del norte del país ${ }^{5}$ cuyas principales empresas pertenecían a capitales estadounidenses (Godoy, 2017). Esta región árida carecía de alimentos y agua suficiente para el riego. Los períodos de sequía y la escasez de suministros paralizaban la actividad minera, puesto que el personal recibía escasa alimentación. Su precio era muy alto debido a los costos de transporte desde el sur del país. Por ello, las empresas mineras se veían obligadas a aumentar los salarios, reduciendo así sus ganancias (Ortega Martínez, 2014). Frente a esta limitación, los valles fértiles de las regiones de Atacama y Coquimbo representaron la única solución para cultivar alimentos y suministrar los sitios mineros a bajo costo.

Fondos consultados: Archivo Nacional de Chile. DOH: 534, 1952; 628, 1955. MOP, 1969, 7357; 6640, 1963; 8712, 1984. Memorias del MOP 1976- 
Esta situación condujo al estado chileno a llevar a cabo la reforma agraria en 1967 y a apoyar la agricultura para mantener una fuerza laboral local a pesar de la aridez del clima. Desde la década de los años 1940, la Corporación de Fomento otorgó préstamos a grandes terratenientes para que extendiesen sus cultivos usando las aguas del embalse de La Laguna (1945) y la red de canales existente. Mas tarde, en 1949, el Departamento de Obras Públicas financió el desecamiento de los humedales costeros de La Serena para permitir el cultivo de la planicie costera. Además, el estado construyó tanques individuales para los grandes terratenientes. En el contexto de urbanización incipiente del valle, esto permitió reducir la competencia con la demanda de agua para consumo doméstico. En 1949, las redes de agua potable de La Serena, de Coquimbo y de Vicuña fueron renovadas y ampliadas. Finalmente, como parte de la reforma agraria (1967 - 1973), el estado instaló estanques colectivos en distintos asentamientos.

\section{La extensión de las redes de agua potable: el auge del capitalismo agrícola y minero (1973 - 1990)}

El golpe militar en 1973 marcó un punto de inflexión económico y político en el país. Debido a las estrategias de los inversionistas norteamericanos y a las nuevas orientaciones económicas monetaristas de la Junta militar (Rinke, 2013), los sectores agrícolas y mineros del valle se insertaron gradualmente en los mercados mundiales. En 1974, el estado promulgó una contrarreforma agraria que impulsó un capitalismo agrario (Kay, 1991) al otorgar títulos de propiedad privada a pequeños agricultores y créditos condicionados al desarrollo del monocultivo de uvas. Se buscaba convertir a los campesinos en empresarios agrícolas (Bengoa, 1983). Por otro lado, el estado explotó la mina de oro El Indio antes de transferirla al capital extranjero, promoviendo así la internacionalización del sector minero.

Durante este período, las instituciones estatales se centraron más en la definición de un plan estratégico y un marco regulatorio hídrico que en la realización de inversiones en objetos técnicos hídricos. Las contribuciones financieras del estado fueron pocas. Se limitaron a los proyectos realizados a partir de 1986 por la CNR en virtud de la aplicación de la ley de riego. En el valle de Elqui, esta ley financió 16 proyectos, de los cuales 12 eran destinados a grandes agricultores que concentraban $83 \%$ de las ayudas. Las inversiones realizadas se focalizaron principalmente en los servicios de agua potable y de saneamiento. Por un lado, el Servicio Nacional de Obras Sanitarias financió la extensión de las redes de agua de La Serena, de Coquimbo y de Vicuña e instaló, en 1984, una planta de tratamiento en La Serena. Por otro lado, en el marco del programa de agua potable rural, el SENDOS construyó, con el apoyo del Banco Interamericano de Desarrollo (BID), los pozos y estanques de 20 Comités de agua potable rural.

\section{La búsqueda de la eficiencia para satisfacer la creciente demanda de agua (1990 - 2018)}

Desde la transición a la democracia comienza una financiarización de la economía y la integración del país en la globalización (Ffrench-Davis, 2004). La inversión de capital extranjero en los sectores agrícola, minero e inmobiliario conduce a una incorporación del valle en el comercio mundial. En primer lugar, se desarrolla una agricultura comercial con la iniciativa de empresas agroindustriales o pequeños y medianos empresarios agrícolas. Aguas abajo del embalse Puclaro, 
se cultivan legumbres y frutas, alimentados en agua mediante la construcción de pozos subterráneos y la extensión de los canales, destinados al mercado interno. Aguas arriba, se cultivan frutas y particularmente uvas de mesa exportadas hacia los Estados Unidos de América. Además, la producción minera aumenta durante este período actual. Junto a la mina El Indio, el capital extranjero invierte en las minas de Talcuna, Lambert, San Gerónimo y Andacollo, cuyos proyectos se extienden. En fin, la especulación inmobiliaria produce una expansión urbana impulsada por el auge del turismo (Rehner \& Rodríguez-Leiva, 2017). Los nuevos centros comerciales revelan la incorporación por los habitantes de modelos de consumo occidentales u ostentosos. Los malls se encuentran principalmente en el litoral. En un contexto de privatización de los servicios primarios (agua, electricidad, transporte, educación), que afecta a todo el país (Gárate, 2012), el acceso al crédito al consumo se vuelve esencial para los hogares.

Este punto de inflexión en la economía política se basa en un crecimiento exponencial de las inversiones en objetos técnicos hídricos. En 1999, fue inaugurado el embalse Puclaro, acumulando más de $200000 \mathrm{~m}^{3}$ de agua. Para crear un sistema eficiente de distribución de agua, la CNR y el Departamento de Obras Públicas invirtieron más de 10 millones de pesos en la instalación de tanques individuales y de sistemas de riego por goteo. Si esta ayuda se otorgó principalmente a los grandes agricultores, a partir del año 2016, la CNR financió planes piloto destinados a lo que llamó «pequeños agricultores y pueblos originarios». La Junta de vigilancia del Río Elqui también ayudó a renovar los sistemas de riego instalando compuertas automáticas en la entrada de los canales estratégicos, ubicados aguas abajo del embalse, donde se consolida la agricultura comercial.

Conjuntamente, la extensión de las redes de agua y de saneamiento ha atraído a inversionistas inmobiliarios. Desde el año 2003, la empresa de agua potable, Aguas del Valle S.A, ha aumentado las conexiones de agua y saneamiento en los hogares ayudada por la construcción de pozos y de plantas de tratamiento en Vicuña y en Paihuano. Además, el Ministerio de Obras Públicas financió la infraestructura básica de 24 Comités de agua potable rural y varios sistemas de tratamiento de aguas residuales.

\section{Los mercados de aguas en la cuenca del rio Elqui}

Los usuarios del valle utilizan estratégicamente los objetos técnicos hídricos y el derecho para adquirir derechos de agua o volúmenes de agua. Estas prácticas conducen a la construcción social de cinco mercados de agua en la cuenca (Figura $\mathrm{N}^{\circ} 3$ ). 
Figura $\mathrm{N}^{\circ} 3$.

Los mercados del agua en la cuenca del río Elqui

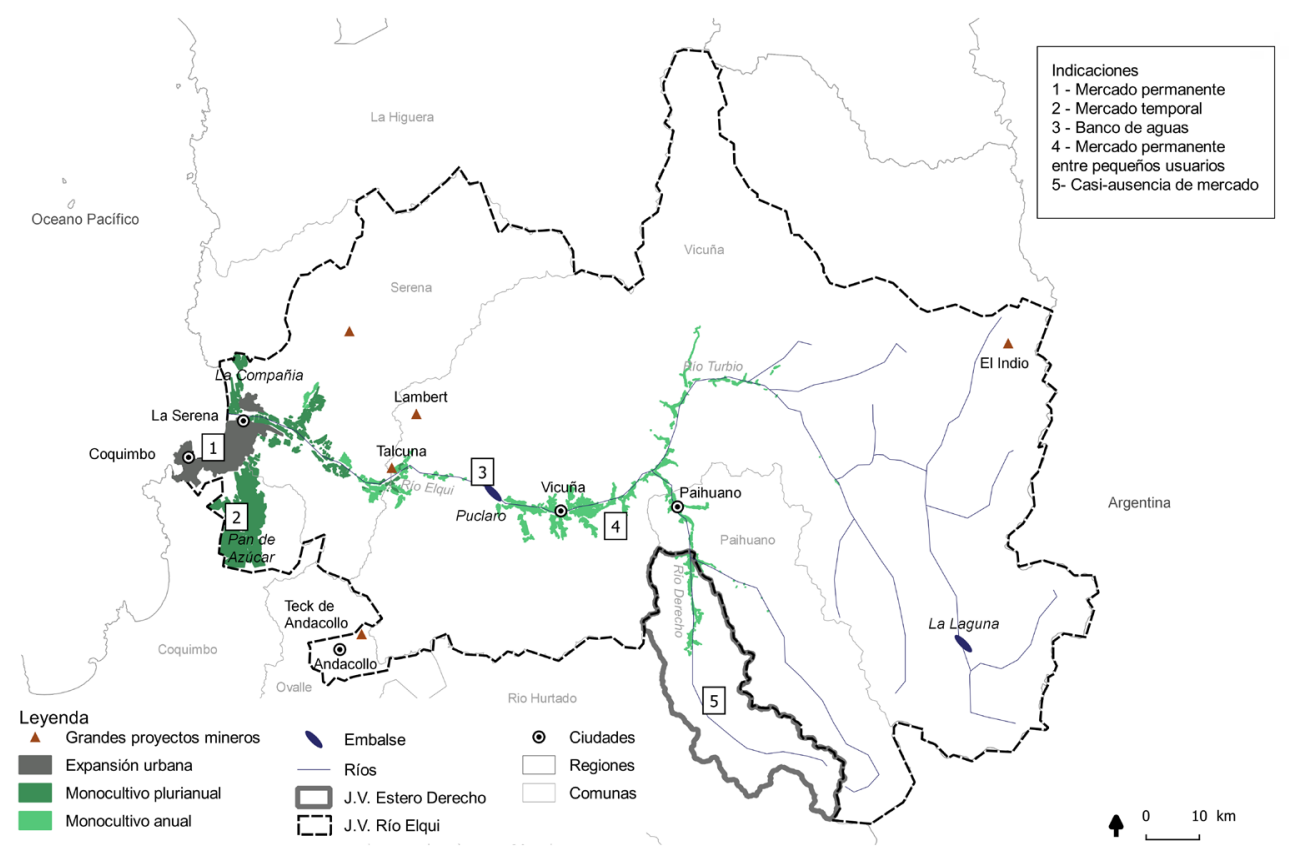

Fuente: Elaboración propia.

\section{Expansión urbana de La Serena-Coquimbo: mercado permanente y banco de aguas}

En la desembocadura del río Elqui, las prácticas de las empresas inmobiliarias y la empresa de agua potable producen dos tipos de mercados de agua: un mercado permanente y un banco de aguas. La compra de derechos y volúmenes de agua de estos actores contribuye a la expansión urbana de la conurbación La Serena-Coquimbo. Por un lado, en el mercado permanente, los principales compradores de derechos son sociedades anónimas, vinculadas a empresas inmobiliarias. Antiguos terratenientes, campesinos o titulares de derechos de agua, llamados «mercedes de agua», otorgados por el estado antes de la aplicación del primer Código de aguas de 1951, les vendieron sus derechos de agua. A menudo son «derechos de papel» debido al cambio del uso del suelo producido por la urbanización que inhabilitó paulatinamente los canales de riego. Estos intercambios producen un mercado permanente puesto que están registrados en los registros de la propiedad del CBR de La Serena. Después, las empresas inmobiliarias transfieren los derechos de agua a la empresa de agua potable a cambio de una extensión de la red de distribución y saneamiento hacia la periferia donde se ubican sus proyectos inmobiliarios.

Por otro lado, la empresa de agua potable compra volúmenes de agua a la Junta de vigilancia del Río Elqui. El gerente de la empresa me explica los diferentes acuerdos con esta organización: 
"Con el crecimiento de La Serena-Coquimbo todos se defienden como pueden. Como tampoco hay prioridad para el consumo humano [...] Entonces entramos a competir con las compañías mineras que ofrecen un precio que no podemos pagar. Este es el principal problema que surge aquí [...] Pero sí, tenemos una buena relación con la Junta de vigilancia. De hecho, teníamos, hoy tenemos un contrato con ella, según el cual nos da el agua que tiene disponible. Nos la entregan. Entonces nos dio [...] nos sirvió bien [...] para superar la sequía también" (Gerente de la empresa sanitaria, 2017)

Este extracto de entrevista muestra cómo la empresa de agua potable está en competencia con las compañías mineras para comprar volúmenes de agua a la Junta de vigilancia. La existencia de un contrato y de una rivalidad para comprar derechos de agua revela que el rol de la Junta de vigilancia en la distribución del agua excede el reparto de los derechos de agua asignados por la DGA. Por lo tanto, la Junta de vigilancia actúa como un banco de aguas privado, es decir como "a central institution that acts as a clearinghouse for users who wish to purchase or sell" (Hadjigeorgalis, 2009: 52).

Desde la declaración de áreas de prohibición en la cuenca por parte de la DGA, la Junta de vigilancia juega con la figura legal de "derechos de agua a carácter eventuales», reconocida en el Código de aguas, para vender volúmenes de agua. Formalmente, esta figura legal se refiere a la posibilidad que tiene la DGA para otorgar derechos de agua de manera excepcional en una cuenca sobreexplotada. En tiempos normales, los usuarios solicitan estos derechos a la DGA. En este caso, los usuarios los adquieren mediante la firma de contratos con la Junta de vigilancia para suscribirse a lo que han llamado «derechos Puclaro», que oficialmente permiten a la Junta reembolsar el embalse al Estado. Por lo tanto, la Junta se apropia de una categoría jurídica nacional para vender volúmenes de agua a actores ubicados aguas abajo del embalse. La creación de estos «derechos Puclaro» revela la producción localizada de reglas jurídicas por la Junta de vigilancia y los actores para vender o adquirir derechos de agua.

\section{La agricultura comercial en el mercado temporal}

Aguas abajo del embalse, la formación de un mercado temporal informal o Spot Market (Hadjigeorgalis, 2004) contribuyó al desarrollo de la agricultura comercial. Los dueños de derechos de agua los arriendan informalmente. El inquilino tiene derecho a usar cierta cantidad de agua por un tiempo limitado. Las transacciones se realizan principalmente entre los empresarios agrícolas que han beneficiado del mejoramiento de los canales de riego y de la instalación de compuertas automáticas. Estos objetos técnicos hídricos facilitan la transferencia de volúmenes de agua. Los arrendamientos de agua revelan la existencia de acuerdos legales informales entre agricultores que comparten una misma sección de canal, especialmente en los sectores de Pan de Azúcar y La Compañía, donde la expansión de la agricultura se ha logrado con la ayuda de la extensión de los canales en arborescencia. Este mercado temporal sostiene el auge de la agricultura, puesto que los empresarios cultivan plantaciones en función de los pronósticos estacionales y los precios de venta. En este sentido, las transacciones revelan una apropiación de los objetos técnicos hídricos y la realización de arreglos jurídicos informales con el objetivo de adquirir agua para ajustar la producción de cultivos cuatrienales a la demanda del mercado nacional. 


\section{Los proyectos mineros: adquisición de títulos en el banco de aguas}

La expansión de los proyectos mineros de San Gerónimo, Talcuna y Teck de Andacollo, ubicados aguas abajo del embalse, se sostiene en la compra de volúmenes de agua a la Junta de vigilancia. Al igual que la empresa de agua potable, las empresas mineras pueden obtener volúmenes de agua firmando un contrato con la Junta de vigilancia de Rio Elqui. El jefe de la Unidad Ambiental de la empresa minera de San Gerónimo confirma su existencia sin querer revelar su contenido:

"Jefe de Unidad: Para trabajar, necesitamos 60 litros por segundo. Como reciclamos el 75 y el $80 \%$ del agua en el relave, nuestro uso real del agua del canal Marqueza oscila entre el 20 y el $25 \%$ de los 60 litros por segundo que tenemos, ya que el resto se recupera.

Chloé Nicolas-Artero: - ¿Y qué hacen con el resto?

J: - No! jFluye! Uh [...] No sé, no conozco el contrato con la Junta de vigilancia, así que no sé el destino de las aguas [...] que no se utilizan. ¡Prefiero no responder porque no lo sé! No sé el contenido del acuerdo" (Jefe de la Unidad Ambiental, Minera San Gerónimo, 2018)

Este extracto de entrevista evidencia la existencia de un contrato entre la empresa minera y la Junta de vigilancia. Muestra la racionalización del uso de agua por la empresa que calcula meticulosamente su nivel de consumo. En ese sentido, las empresas de San Gerónimo y Andacollo trabajan con la compañía israelí Netafim para adaptar las innovaciones tecnológicas destinadas a economizar agua en su proceso de producción. El ahorro permite a las empresas arrendar el agua que le sobra en los mercados temporales y así evitar pagar la multa por no usarla. Por lo tanto, la utilización de tecnologías por estas compañías y la creación de reglas localizadas con la Junta de vigilancia apuntan a concentrar el agua y transformarla en un activo financiero colocado en el mercado para aumentar su rentabilidad.

\section{Pequeños titulares en el mercado de aguas permanente}

La mayoría de las compras y ventas de derechos formales registrados en los CBR de La Serena y de Vicuña se realizan entre pequeños titulares de derechos de agua (Figura $N^{\circ} 1$ ). A partir de la consulta de los registros de los CBR y de las entrevistas, los defino como personas físicas que poseen e intercambian, entre ellas, fracciones de acciones de agua, distinguiéndose de las personas jurídicas. El agua se destina al riego de las parcelas o de los huertos o al uso recreativo y paisajístico de un turismo emergente. Los usuarios se concentran en los canales más antiguos ubicados principalmente aguas arriba del embalse Puclaro. Los intercambios conciernen principalmente los titulares de un mismo canal, puesto que reduce los costos de transacción vinculados al traspaso de un derecho de agua de un canal a otro.

La venta de agua es parte de una estrategia de supervivencia de los usuarios que usan sus acciones como una cartera de activos. Las venden según sus necesidades económicas y sociales. Las ganancias obtenidas son utilizadas para pagar el acceso a la salud, al alojamiento, a la educación o para rembolsar una deuda. El presidente de la Junta de Vigilancia del Río Derecho explica estas razones:

“Por necesidad y porque: 'Bueno, yo tengo tres acciones' y 'Pucha necesito y vendo una', o porque: 'Yo vendo una acción pucha porque necesito comprar un auto'; o 'Necesito porque 
pucha estoy pillado entonces pucha ofrezco y me la compran"” (Presidente de la Junta de Vigilancia, 2017)

Esta situación no puede entenderse sin considerar el contexto económico nacional establecido desde el golpe de estado caracterizado por la privatización de los servicios básicos y la deuda de los hogares (Moulian, 2002).

La compra de fracciones de acciones por pequeños usuarios se explica por su deseo de adquirir agua para mantener un acceso mínimo al recurso. Muy a menudo, estas compras representan una cantidad irrisoria de agua, porque los usuarios no entienden la correspondencia entre la unidad de medida impuesta por el Estado y la Junta de vigilancia, en litros por segundo, y la medida utilizada en los turnos de agua del canal - en horas o en cuadras-. Estas compras también ponen de manifiesto la desesperación de los habitantes ante el bajo caudal de agua recibido debido a la aplicación del desmarque - un porcentaje de la acción asociada a un derecho de agua distribuido e impuesto por la Junta de vigilancia -, a robos o incluso a pérdidas por infiltración o por evaporación.

La adquisición de derechos aumenta la posibilidad de obtener un préstamo bancario, como lo muestra el registro de hipotecas de agua en los CBR (Figura $N^{\circ} 1$ ). Los derechos de agua se convierten en una garantía bancaria más importante que los bienes inmuebles, puesto que su valor excede el de los terrenos y de los bienes inmobiliarios. Por lo tanto, la inserción de los pequeños usuarios en el mercado de aguas se explica por razones financieras vinculadas al contexto de la economía política nacional y por la precarización de su acceso al agua.

\section{La cuenca del río Derecho: casi-ausencia de mercado de aguas}

El directorio de la Junta de vigilancia del Estero Derecho impide la construcción social del mercado de aguas. En este valle, ubicado aguas arriba del embalse Puclaro, las empresas agrícolas buscan adquirir derechos y los pequeños agricultores quieren vender o alquilar los suyos. Desde 1948, la Dirección General de Aguas ha declarado la cuenca sobreexplotada razón por la cual no otorga nuevos derechos de agua. Por lo tanto, la adquisición de nuevos derechos solo se puede realizar mediante la compra de tierras - si los derechos de agua están vinculados a ellas - o en el mercado de aguas - si los derechos están regularizados.

Según el gerente de la Junta de vigilancia del Estero Derecho, la existencia de un mercado de aguas en el valle representa una amenaza para los habitantes y su organización. Le preocupa el futuro de los pequeños agricultores que necesitan agua para mantener sus actividades y sus huertos de subsistencia. Además, la concentración de derechos por parte de las empresas agrícolas puede permitirlas obtener la mayoría de los votos durante las elecciones del directorio en la Junta y facilitarlas su control. Durante una entrevista, el gerente se exclama: “¡Estas personas ya tienen prácticamente más del $40 \%$ de las acciones del río!» iSolo los Luksic!"6 (Gerente de la Junta de vigilancia, 2017).

El entrevistado se refiere aquí a las empresas agrícolas cuyo capital es controlado por Andrónico Luksic, uno de los empresarios mas importantes de Chile, y sus familiares 
La concentración de votos otorgaría un mayor poder a las empresas agrícolas que tendrían derecho de influir o dictar las reglas de distribución del agua en la Junta. Para mantener el control de la organización y la mayoría de los votos a su favor, el gerente disuade a los pequeños usuarios de vender sus derechos:

“iPero se hacen daño a ellos mismos porque su caudal disminuye! [...] Aparte de eso, tienen que estar en el mismo canal. Aunque el Código de aguas permita transferir acciones de un canal a otro, se tiene que hacer un nuevo estudio. Primero tienes que pedir permiso a los agricultores para reducir o subir el agua del canal. Ellos seguramente dirán que sí, pero también, tienes que eliminar los litros por segundo al otro canal. Y tienes que hacer un estudio con un ingeniero y cambiar el escurrimiento crítico. La transferencia de acción tiene un costo muy alto. Porque vienen a verme: 'Don Juan, quiero venderle agua a Luksic, pero estoy en el Canal del Arenal y él en el Canal de Jarilla'. Yo les digo: «ipuedes hacerlo, pero verás el costo! (Gerente de la Junta de vigilancia, 2017).

Por lo tanto, el gerente de la Junta desanima a los usuarios de vender sus derechos invocando las dificultades jurídicas y materiales para realizar las transacciones, subrayando especialmente los costos de estas últimas. Su objetivo es convencer los pequeños agricultores de no vender sus derechos, como lo explica uno de ellos:

"Sí, el agua se puede vender. Pero [...] depende de los canales, ¿eh? ¡Depende de los canales! Yo tenía 6 acciones en el canal Molino y quería venderlas, pero no le interesaba a nadie... porque no es un canal muy comercial... Y quise bajarlas al canal Jarilla, pero de nuevo, el presidente de la Junta me dijo que era un poco complicado, que no era fácil, tenía que ser con un abogado, y que una cosa y que otra. iBueno! Tengo agua y quería venderla, pero no pude. También le pregunté al gerente si había una posibilidad y hasta ahora no me ha contestado" (Pequeño agricultor de Pisco Elqui, 2017)

En este caso específico, las relaciones de poder entre los dirigentes de la Junta de vigilancia y las empresas agrícolas explican la casi inexistencia de transacciones formales de derechos de agua.

\section{Discusión}

Este artículo se distingue de la abundante literatura que presupone la existencia de un mercado de aguas en Chile desde la promulgación del Código de 1981 (Panez-Pinto et al., 2017; Romero et al., 2012). A diferencia de estas investigaciones, en este artículo se estudian empíricamente, mediante un trabajo etnográfico, de entrevistas, y la consulta de archivos, las condiciones para la formación de intercambios mercantiles formales o informales de agua. Desde la geografía legal crítica, se aportan elementos a la reflexión sobre la construcción de los mercados de agua, y por ende, sobre la propiedad del agua, enfatizando sus dimensiones legales y espaciales. A partir del análisis de las transacciones, identificamos la formación de cinco tipos de mercados de agua en una cuenca. Los mercados se estructuran en dos etapas. En un primer tiempo, la instalación de distintos objetos técnicos hídricos en la cuenca, desde la segunda mitad del siglo XX, atraen nuevos inversionistas en el valle transformando su economía política. En un segundo tiempo, estos 
inversionistas y los habitantes se apropian de los objetos técnicos hídricos, y del derecho, para comprar o vender agua, produciendo distintos tipos de mercados de agua.

El artículo propone además una reflexión sobre la propiedad del agua y su reparto a nivel de la cuenca. Muestra como la definición de la propiedad sobre las aguas y la formación de un mercado no dependen únicamente de su reconocimiento como bien privado en la Constitución política del Estado. La existencia de una pluralidad de mercados de agua, y a veces su ausencia, revela como la propiedad sobre las aguas es definida localmente por los usuarios en función de su uso y sus intereses económicos. El reparto del agua, a nivel de la cuenca, está fuertemente determinado por las intervenciones de las Juntas de vigilancia, quienes, según algunos juristas, podrían tener en Chile el potencial de aplicar una "Gestión Integrada de los Recursos Hídricos". Si según el Código de aguas, estas organizaciones deben únicamente velar en el reparto de las aguas superficiales de la cuenca respetando los derechos de agua de cada usuario, el estudio de caso revela el poder de estas organizaciones en la asignación preferencial del agua a empresas agrícolas y sanitarias (caso de la Junta de vigilancia del rio Elqui) o en la construcción de resistencias a la apropiación por empresas agrícolas (caso de la Junta de vigilancia del Estero derecho). Estas organizaciones tienen el poder de definir el goce de las aguas, desvinculado de la tenencia de los títulos formales.

Al fin, el artículo constituye una crítica a la nueva economía de recursos y los discursos neoliberales sobre el agua (Vergara Blanco 2015; Thobani, 1997). El reconocimiento de la propiedad privada y el mercado de aguas debería incentivar la inversión privada en infraestructura de riego, un uso racional del agua y el desarrollo de los sectores productivos. Las observaciones de campo muestran que el reconocimiento jurídico de la propiedad privada sobre las aguas no explica la aparición del mercado de aguas ni el desarrollo económico de estos sectores. Por el contrario, destaca el rol de los objetos técnicos hídricos financiados por el Estado, y la ayuda multilateral, en la producción del espacio, así como en la de la redefinición de reglas locales. Estos aspectos materiales, espaciales y jurídicos son los que inciden en la formación de los mercados de agua.

\section{Conclusión}

El presente artículo ha mostrado el rol de los objetos técnicos hídricos en la construcción social de los mercados de agua. Los usuarios utilizan estos objetos, producto de inversiones realizadas desde la segunda mitad del siglo XX, así como instrumentalizan el derecho para comprar o vender agua. Estas reapropiaciones conjuntas del espacio y del derecho, se distinguen en función del tipo de usuarios y de su localización en la cuenca, explicando así la formación de mercados de agua que se diferencian espacial y jurídicamente. El artículo identifica y categoriza cinco mercados de agua en la cuenca del río Elqui que producen una dinámica espacial singular en la cuenca. La expansión inmobiliaria y urbana de la Serena-Coquimbo se sostiene gracias a la existencia de un mercado permanente y la intervención de la Junta de vigilancia del río Elqui que opera como un banco de aguas. En el sector de Pan de azúcar, los empresarios agrícolas arriendan derechos de agua en el mercado temporal para desarrollar una agricultura comercial. Las empresas mineras adquieren los derechos de agua que son ofrecidos por la Junta de vigilancia. La mayoría de los pequeños usuarios localizados aguas arriba del embalse con un acceso al agua y condiciones de vida precarias se insieren en un mercado permanente buscando mejorar su situación. Al fin, en la subcuenca del Estero Derecho, la Junta de vigilancia del Estero derecho frena la venta de dere- 
chos de los pequeños agricultores a la empresa agrícola local perteneciente a Andrónico Lucksic para evitar la formación de un mercado permanente.

La geografía legal presenta una contribución metodológica y epistemológica en el análisis de los mercados de agua. Por una parte, invita a analizar empíricamente el funcionamiento del mercado de aguas mediante el estudio del derecho en acción. La mercantilización y privatización de las aguas deben ser discutidas en base a observaciones empíricas sobre procesos concretos de definición de la propiedad sobre las aguas y su reparto local y cotidiano. Por otra parte, esta corriente de la geografía ofrece una contribución epistemológica al integrar la materialidad, la espacialidad e historicidad en el análisis de los mercados de agua. La propiedad sobre las aguas no es definida únicamente por los títulos formales otorgados o adquiridos. Se concretiza materialmente mediante el uso de objetos técnicos hídricos. Por ello, su materialización no es homogénea en el espacio, sino que toma formas distintas en función de la localización del punto de captación en la cuenca. Al fin, la propiedad sobre las aguas es cambiante. Se transforma en el tiempo, puesto que es definida espacialmente y que el espacio es transformación, producida, entre otros, por la economía política y por las relaciones de poder que definen el acceso al agua. Tal como lo sostiene Nicolas Blomley el agua se puede calificar de splice (empalme), en el sentido que es un objeto cuyas características jurídicas y espaciales son indisociables. Las características jurídicas del agua dependen de su localización y su forma, y a su vez, las características espaciales del agua pueden ser modificadas por el derecho (City, 2010).

El artículo contribuye a la geografía jurídica al destacar la dimensión espacial de la propiedad. Tomando el ejemplo del agua, representa un aporte mostrando como se definen los derechos de propiedad sobre un elemento fluido y dinámico como el agua. Las reflexiones desde la geografía jurídica sobre la propiedad han subrayado como esta es definida por procesos de territorialización que establecen fronteras materiales y simbólicas entre lo privado y lo público. La propiedad y el territorio se constituyen mutuamente. La propiedad es un medio por el cual son creados y reconstruidos lugares. Se basa en un proceso particular de espacialización, donde sujeto y objeto son separados e individualizados y donde los sujetos tienen una "cultura de la propiedad" especifica (Blomley, 2003; 2004; 2005; 2016). Estas visiones tienden a homogeneizar y totalizar la propiedad y el territorio impidiendo un estudio detallado de la pluralidad de relaciones sociales, en particular las negociaciones y resistencias legales que definen la propiedad. Siguiendo a Ripoll y Veschambre (2005), el uso de las nociones de frontera y de territorio para definir la propiedad aparecen como una forma de espacialismo ya que separan la aprehensión de lo social y lo espacial y transmite una representación globalizante del mundo social al negar las relaciones sociales de poder que cruzan las relaciones con el espacio. Siguiendo los trabajos que han mostrados que los límites de la propiedad no son estables (Forest, 2009)the province of Québec has established many provincial parks (known as national parks since 2002 el presente artículo subraya la dimensión espacial de la propiedad destacando dos aristas. Por una parte, la propiedad sobre las aguas se define por relaciones multiescalares. El derecho de aguas se define en distintos niveles de acción, de forma compleja y no por aplicación unilateral del artículo 19 de la Constitución o del Código de aguas. El mercado de aguas emerge tras la realización de arreglos y negociaciones entre múltiples actores situados en distintas escalas. Por otra parte, como lo hemos mostrado, las transacciones de derechos de agua se basan en una instrumentalización conjunta e indisociable de las reglas jurídicas y del espacio. La apropiación del derecho y de los objetos técnicos hídricos, en un espacio dado, es indispensable para transferir derechos de agua y concretizar la propiedad 
sobre las aguas. Por lo tanto, la propiedad del agua tiene una dimensión material y legal indisociable y se define localmente. Es parte de un proceso geohistórico de producción conjunta del espacio y del derecho que revela la dimensión espacial del derecho.

\section{Referencias}

BAUER, C. Bringing Water Markets down to Earth: The Political Economy of Water Rights in Chile, 1976-1995. World Development, 1997, Vol. 25, N05, p. 639656. https://doi.org/10.1016/S0305750X(96)00128-3

BAUER, C. Contra la corriente: privatización, mercados de agua y el Estado en Chile, Santiago de Chile, LOM Ediciones, Colección ecología y medio ambiente, 2002.

BAUER, C. Results of Chilean Water Markets: Empirical Research since 1990: results of chilean water markets. Water Resources Research, 2004, Vol. 40, No9, p. 1-11. https://doi.or$\mathrm{g} / 10.1029 / 2003$ WRO02838

BAUER, C. Canto de sirenas: el derecho de aguas chileno como modelo para reformas internacionales. Santiago de Chile: Ediciones El Desconcierto, 2015.

BENGOA, J. El campesinado chileno después de la reforma agraria. Santiago: Ediciones Sur, 1983.

BJORNLUND, H. Efficient Water Market Mechanisms to Cope with Water Scarcity. International Journal of Water Resources Development, 2003, Vol.19, N4, p.553567. https://doi. org/10.1080/0790062032000161364

BLOMLEY, N. Law, Space, and the Geographies of Power. New York: Guilford Press, 1994.

BLOMLEY, N. Law, Property, and the Geography of Violence: The Frontier, the Survey, and the Grid. Annals of the Association of American Geographers, 2003, Vol.93, N01, p. 121-141.https:// www.jstor.org/stable/1515327

BLOMLEY, N. Flowers in the bathtub : Boundary crossings at the public-private divide. Geoforum, 2005, Vol. 36, N³, p. 281-296. https://doi.org/10.1016/j.geoforum.2004.08.005

BLOMLEY, N. Cuts, Flows, and the Geographies of Property. Law, Culture and the Humanities, 2011, Vol.7, No2, p. 203216.https://doi.org/10.1177/1743872109355583

BLOMLEY, N. The Boundaries of Property : Complexity, Relationality, and Spatiality Law \& Society Rev Law \& Society Review, 2016, Vol. 50, No1, p. 224255. https://doi.org/10.1111/lasr.12182

BLOMLEY, N. The territory of property. Progress in Human Geography, 2016, Vol.40, N05, p. 593609.https://doi.org/10.1177/0309132515596380 
BRAVERMAN, I.; BLOMLEY, N.; DELANEY, D. \& KEDAR, A. The expanding spaces of law: a timely legal geography. Stanford: Stanford University Press, 2014.

BUDDS, J. La demanda, evaluación y asignación del agua en el contexto de escasez: un análisis del ciclo hidrosocial del valle del río La Ligua, Chile. Revista de Geografía Norte Grande, 2012, Vol. 52, p. 167184. http://dx.doi.org/10.4067/s0718-34022012000200010

BRISCOE, J.; ANGUITA, P. \& PEÑA, H. Managing Water as an Economic Resource: Reflections on the Chilean Experience. Environ. Dep, World Bank, Washington, 1998, D. C, n62

CALLON, M. Éléments pour une sociologie de la traduction : la domestication des coquilles Saint-Jacques et des marins-pêcheurs dans la baie de Saint-Brieuc, L'Année sociologique (1940/1948), 1986, Vol. 36, p. 169-208.

CASADO-PEREZ, V. The Role of Government in Water Markets. London: Routledge, 2016.

CITY, C. La géographie du droit, l'eau et l'environnement. En : FOREST, P. (eds.). Géographie du droit : Épistémologie, développement et perspectives. Québec, Presses Université Laval, 2009, p. 157-176.

DONOSO, G.; MONTERO, J-P. \& VICUNA, S. Análisis de los mercados de derechos de aprovechamiento de agua en las cuencas del Maipo y el sistema Paloma en chile: efectos de la variabilidad en la oferta hídrica y de los costos de transacción. Revista de derecho administrativo económico, 2000, Vol 3, No2, p. $367-387$.

DOUROJEANNI, A. \& JOURAVLEV, A. El Código de aguas de Chile: entre la ideología y la realidad. Serie Recursos naturales e infraestructura, CEPAL, 1999, No3, p.1-84

EASTER, W.; ROSEGRANT, M. \& DINAR, A. Formal and Informal Markets for Water: Institutions, Performance, and Constraints. The World Bank Research Observer, 1999, Vol.14, No 1, p.99116.

FFRENCH-DAVIS, R. Entre el neoliberalismo y el crecimiento con equidad: tres décadas de política económica en Chile. Buenos Aires, Siglo XXI, 2004.

FOREST, P. Empiètements et stratégies géo-légales : Le cas de la coupe en mosaïque en bordure des parcs nationaux du Québec. Canadian Geographer, 2009, Vol.53, No2, p. 191-207. https://doi. org/10.1111/j.1541-0064.2009.00253.

GÁRATE CHATEAU, M. La revolución capitalista de Chile: 1973-2003. Santiago de Chile: Ediciones Universidad Alberto Hurtado, 2012.

GARRICK, D.; HERNÁNDEZ-MORA, N. \& O'DONNELL, E. Water Markets in Federal Countries: Comparing Coordination Institutions in Australia, Spain and the Western USA. Regional Environmental Change, 2018, Vol.18, No6, p.15931606. https://doi.org/10.1007/s10113-018-1320-z

GERMAINE, M-A. ;BLANCHON, D.; TEMPLE-BOYER, E. \& FOFACK-GARCIA, R. (2019). Les objets techniques au prisme du cycle hydrosocial : renouveaux théoriques et empiriques. Développement durable et territoires, Vol. 10, No 3 https://doi.org/10.4000/developpementdurable.16287 
GODOY ORELLANA, M. Mundo minero y sociabilidad popular en el norte chico. Chile, 1780-1900. Santiago de Chile, Ediciones Mutante, 2017.

GRAFTON, R. Q.; LIBECAP, G.; MCGLENNON, S.; LANDRY, C. \& O'BRIEN, B. An Integrated Assessment of Water Markets: A Cross-Country Comparison. Review of Environmental Economics and Policy, 2011, Vol.5, No 2, p.21939. https://doi.org/10.1093/reep/rer002

HADJIGEORGALIS, E. Comerciando con incertidumbre: los mercados de agua en la agricultura chilena. Cuadernos de economía, 2004, Vol.41, No 122, p.3-34. https://www.jstor.org/stable/41951506

HADJIGEORGALIS, E. A Place for Water Markets: Performance and Challenges. Review of Agricultural Economics, 2009, Vol.31, No 1, p. 5067. https://www.jstor.org/stable/30224846

HEARNE, R. \& EASTER, K. The Economic and Financial Gains from Water Markets in Chile. Agricultural Economics, 1997, Vol.15, No 3, p.18799. https://doi.org/10.1111/j.1574-0862.1997.tb00431.x

HOLDER, J. \& HARRISON, C. (dir.) Law and Geography, Oxford: Oxford University Press, 2003.

HOWITT, R. Empirical analysis of water market institutions: the 1991 California water market. Resource and energy economics, 1994, Vol. 16, p.357371. https://doi.org/10.1016/09287655(94)90026-4

KAY, C. Agrarian policy and democratic transition in Chile: continuity or change? Working paper series, 1991, Vol.101, N01, p.125. https://doi.org/10.1016/S0261-3050(96)00012-5

LATOUR, B. Nous n'avons jamais été modernes. Essai d'anthropologie symétrique. Paris : La Découverte, 1991.

MOLLE, F. Defining Water Rights: By Prescription or Negotiation? Water Policy, 2004, Vol.6, N³, p. 20727. https://doi.org/10.2166/wp.2004.0014

MOULIAN, T. Chile actual: anatomía de un mito. Santiago de Chile: Lom Ediciones, 2002.

OBERTREIS, J.; MOSS, T.; MOLLINGA, P. \& BICHSEL, C. (2016). Water, Infrastructure and political rule : Introduction to the special issue. Water Alternatives, Vol. 9, No2, p. 168-181.

ORTEGA MARTíNEZ, L. La crisis de la minería del Norte Chico, Chile en la primera mitad del siglo XX y la decadencia de la Región de Coquimbo. Nuevo mundo mundos nuevos, 2014, p.1-25. https:// doi.org/10.4000/nuevomundo.67244

PANEZ-PINTO, A.; FAÚNDEZ-VERGARA, R. \& MANSILLA-QUIÑONES, C. Politización de la crisis hídrica en Chile: Análisis del conflicto por el agua en la provincia de Petorca. Agua y Territorio, 2017, Vol.10, p. 131148. https://doi.org/10.17561/at.10.3614

PETIT, O. La nouvelle économie des ressources et les marches de l'eau : une perspective idéologique? VertigO - la revue électronique en sciences de l'environnement, 2004, Vol.5, No2, p.1-10. https://doi.org/10.4000/vertigo.3608 
PRIETO, M. La ecología política del modelo de aguas chileno in Bustos. B., Prieto, M., Barton, J., (eds), Ecología política en Chile. Naturaleza, conocimiento, y Poder. Santiago, Editorial Univeritaria, p.143 - 164, 2015.

PRIETO, M. Transando el agua, produciendo territorios e identidades indígenas: el modelo de aguas chileno y los atacameños de Calama. Revista de Estudios Sociales, 2016, Vol.55, p. 88103.

REHNER, J., RODRÍGUEZ-LEIVA, S. Inversión inmobiliaria en tiempos de auge y crisis: ¿Es la ciudad un producto minero o un derivado financiero? Revista de Geografía Norte Grande, 2017, Vol. 67, p.183210.

REIS, N. Coyotes, Concessions and Construction Companies: Illegal Water Markets and Legally Constructed Water Scarcity in Central Mexico. Water Alternatives, 2014, Vol.7, N³, p. 542-560.

RIOS, M., QUIROZ, J. The Market for Water Rights in Chile: Major Issues. Cuadernos de Economia, 1995, Vol.97, p.317-345. https://www.jstor.org/stable/41951284

RIPOLL, F., \& VESCHAMBRE, V. (2005). Introduction. L'appropriation de l'espace comme problématique. Norois. Environnement, aménagement, société, N0195, p. 7-15.

RINKE, S. Encuentros con el Yanqui: Norteamericanización y cambio sociocultural en Chile (18981990). Santiago de Chile: Ediciones de la Dirección de Bibliotecas, 2013.

ROMERO, H.; MENDEZ, M., \& Smith, P. Mining Development and Environmental Injustice in the Atacama Desert of Northern Chile. Environmental Justice, 2012, Vol.5, N², p. 70 - 76. https://doi. org/10.1089/env.2011.0017

ROSEGRANT, M. \& BINSWANGER, H. Markets in Tradable Water Rights: Potential for Efficiency Gains in Developing Country Water Resource Allocation. World Development, 1994, Vol.22, No 11, p. 613625. https://doi.org/10.1016/0305-750X(94)00075-1

ROSEGRANT, M. \& GAZMURI, R. Reforming water allocation policy through markets in tradable water rights: lessons from Chile, Mexico, and California. Cuadernos de economía, 1995, Vol.32, No97, p. 291315.https://www.jstor.org/stable/41951283

SALIBA, B. Do water markets "work"? market transfers and trade-offs in the southwestern states. Water Resources Research, 1987, Vol. 23, N07, p. 1113-1122.https://doi.org/10.1029/WR023i007p01113

THOBANI, M. Formal Water Markets: Why, When, and How to Introduce Tradable Water Rights. The World Bank Research Observer, 1997, Vol.12, No2, p.161179.https://www.jstor.org/stable/3986407

VERGARA BLANCO, A. Crisis institucional del agua: descripción del modelo, crítica a la burocracia y necesidad de tribunales especiales. Santiago: Thomson Reuters, 2014. 